by energetic particles of heavy ions from surfaces of moons or ring particles (e.g., Saturn). However, these sources are negligible in the Earth's magnetosphere.

\section{Prospects}

Two satellites which are currently contributing major new observations about the composition of the Earth's magnetospheric plasma are the Japanese-US Geotail mission launched in July 1992 into a highly eccentric orbit, and the Swedish-German Freja satellite with an apogee of $1750 \mathrm{~km}$ launched in October 1992. The former will make measurements in the deep tail up to October 1994 before being moved into an orbit with an apogee of $30 R_{E}$ for studies of the near tail. Freja is aimed at investigations of the source region of the magnetospheric ions of ionospheric origin. Geotail will make the first detailed studies of the tail and Freja will carry out for the first time measurements at high resolutions in time using several new instruments.

In the period up to the end of 1995 , four other satellite projects will reach their launch phase (see insert). So there is some hope that several of the open questions will be answered by the end of the decade. On the other hand, we can expect that more detailed measurements of all kinds of plasma and field variables will provide new surprises, thus raising new questions about fundamental properties of the Earth's highly complex magnetospheric plasma system.

[1] Shelley E.G. et al., J. Geophys. Res. 77 (1972) 6104 .

[2] Shelley E.G. et al., J. Geophys. Res. Lett. 3 (1976) 654; Ghielmetti A.G. et al., Geophys. Res. Lett. 5 (1978) 59.

[3] Geiss J. et al., Space Sci. Rev. 22 (1978) 537; Balsiger H. et al., J. Geophys. Res. 85 (1980) 1645 [4] Huiltqvist B., Rev. Geophys. Space Phys. 20 (1982) 589.

[5] Sharp R.D. et al., J. Geophys. Res. 82 (1977) 3324 .

[6] Lennartsson W. \& Sharp R.D., J. Geophys. Res. 87 (1982) 6109.

[7] Young D.T. et al., Adv. Space Res. 1 (1981) 309. [8] Johnson R.G., in "Spacecraft Charging Technology 1980"; Eds: N.J. Stevens \& C. Piek, NASA Conf. Publications CP-2182 (1981) 412.

[9] Lockwood M. et al., J. Geophys. Res. 90 (1985) 4099.

[10] Lennartsson W., J. Geophys. Res. 94 (1989) 3600 .

[11] Lennartsson W., J. Atmos. Terr. Phys. 53 (1991) 1103 .

[12] Lennartsson W. et al., J. Geophys. Res. 90 (1985) 1243.

[13] Gloeckler G. \& Hamilton D.C., Physica Scripta. T18, 73, 1987.

[14] Hamilton D.C. et al., J. Geophys. Res. 93 (1988) 14343.

[15] Ungstrup E. et al., J. Geophys. Res. 84 (1979) 4289; Ashour-Abdalla M.H. \& Okuda H., J. Geophys. Res. 89 (1984) 2235.

[16] Chang T. \& Coppi B., Geophys. Res. Lett. 8 (1981) 1253; Retterer J.M. et al., J. Geophys. Res. 91 (1986) 1609

[17] Temerin M. \& Roth I., Geophys. Res. Lett. 13 (1986) 1109; Lennartsson W., Planet. Space Sci. 28 (1989) 135; Borofsky J.E., J. Geophys. Res. 89 (1984) 2251; Ball L., J. Geophys. Res. 94 (1989) 15257; LeQuéau D. \& Roux A., J. Geophys. Res. 97 (1992) 14929.

[18] Hultqvist B. et al., J. Geophys. Res. 93 (1988) 9765.

[19] Lundin R. \& Hultqvist B., J. Geophys. Res. 94 (1989) 6665

[20] Lundin R. et al., J. Geophys. Res. 95 (1990) 5905 .

\section{Heavy-Ion Therapy at GSI}

Gerhard Kraft from the Gesellschaft für Schwerionenforschung, Darmstadt, describes how new approaches and a novel scanning technique to be implemented at GSI's planned ion-therapy unit will offer biologically efficient, tumour conform treatment.

Beams of heavy charged particles beginning with protons represent the most advanced tools for external, subcutaneous therapy of deep-seated tumours in humans. Compared to electromagnetic radiation, they offer an improved distribution of the dose with depth owing to their small lateral and angular scattering; they travel in virtually straight lines and stop at a definite depth (or "range"). More importantly, the dose profile is inversed in that the energy deposition increases from a plateau with increasing penetration distance up to the Bragg maximum, and then cuts off sharply within a few millimetres. By combining overlapping Bragg peaks it is possible to achieve a homogeneous energy deposition over a defined volume (Fig. 1).

The lateral and range scattering decreases with the square of the atomic number so the precision of beam delivery increases as the atomic number increases. But the nuclear interaction rate also increases yielding lighter nuclear fragments having a longer range than the primary particles. An optimum is reached for ions around carbon where lateral scattering is small and nuclear fragmentation tolerable [1]. Moreover, nuclear fragmentation of ions heavier than protons and helium has strongly forward-peaked reaction kinematics. By measuring the $\gamma$-annihilation

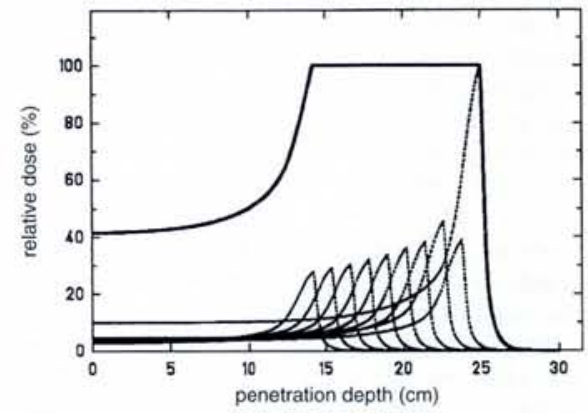

Fig. 1 - The superimposition of Bragg curves to give a uniform dose over a spherical volume. For each Bragg curve, the ionization density rises from a plateau value as the ions slow down, until at the very end of the ion's range the ionic charge is reduced by electron pick-up and the ionization falls rapidly to zero.

quanta of $\beta^{+}$-radioactive ions using positron emission tomography (PET) one can therefore correlate the range distribution with the primary beam in order to localize on-line the beam inside the patient [2].

The increased biological efficiency of heavy ions providing high ionization density in certain types of tumours opens up new dimensions in the treatment of radio-resistant

\title{
HEAVY-ION THERAPY UNIT AT GSI
}

The creation of a heavy-ion therapy unit at GSI (HITAG) was proposed to the German government in May 1993 by the GSI together with the Heidelberg Radiological Clinic and the German Cancer Research Centre, both these institutes having a long tradition in conventional therapies, neutron therapy and the development of advanced treatment techniques. The proposed 13.2 MDM unit (with some 60\% coming from government and other external sources including insurance companies) will consist of a dedicated therapy care and an annex housing a control room, waiting rooms and the like (diagnosis and treatment planning will be in Heidelberg).

The ion beam from the beam line is guided by two dipole magnets and a pair of focussing quadrupoles. Symmetry and achromatism of the beam line is important for safety because the final focus in the patient corresponds to a focus intermediate between the two dipoles. Scintillators used as a beam scratcher at the intermediate focus allow the beam to be controlled nondestructively. Other safety features include an asymmetric layout for the raster scan-

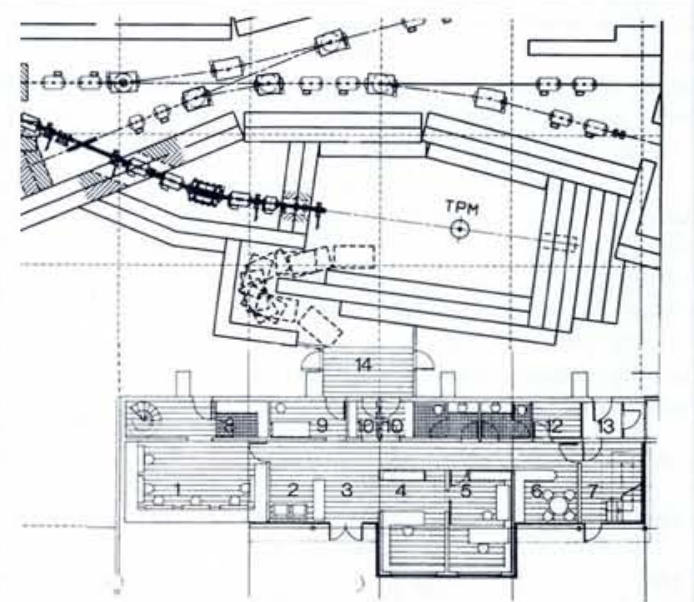

ning magnets so that the beam passes above the patient when the magnets are not powered (in the case of a power failure, the beam will automatically move outside the patient). A highspeed position sensitive counter just in front of the patient will monitor on-line the beam position and compare it with the settings of the scanner magnets in less than a millisecond. Finally, the PET system mentioned in the text will monitor the location of the beam inside the patient.

The cave is under construction and most of the beam-line components have been ordered; construction of the annex starts next year. An extended experimental campaign to characterize carbon and oxygen beams both biologically and physically will allow a choice before the end of 1994. Beam testing in the treatment cave, including exposure to anthropomorphic phantoms, will start in 1995 in preparation for the start of therapy in 1996. The aim is to treat about 100 patients a year suffering from local and inoperable cancers, mostly in the head and neck regions. 
tumours. In conventional therapy and therapy with ions, the rate at which tumour cells are killed is largely governed by the cells' capacity to repair themselves. The most important target for cell inactivation is the DNA molecule: it carries all the genetic information needed for cell functioning. Most of the radiation-induced damage to DNA can be repaired by the cells as long as the individual DNA lesions are well separated. If local damage is increased, the chances of a correct repair are drastically reduced. Comparing the track structures for carbon ions and for protons with the size of the DNA molecule (Fig. 2) shows that carbon is biologically more efficient as it is more likely to produce correlated, unrepairable damage within a single ion track than protons of the same energy.

Ideally, a beam for ion therapy should combine this high biological efficiency in the tumour volume with the creation of most of the repairable damage in the entrance channel in front of the tumour where healthy tissue is present. Recent experiments at GSI have demonstrated that carbon and oxygen ions are very suitable because the biological efficiency increases from a low value in the entrance channel to a four-times higher value at the end of the tumour. But in order to exploit this advantage of ion beams it is essential to adjust the beam so that the range of the particles is restricted to the target volume. This means that strictly conforming the irradiation is the most important requirement in using ions heavier than protons for radiotherapy.

\section{Tumour Conform Irradiation}

In all the particle therapies presently in operation the beam is distributed over the target volume using passive beam-forming elements. These are expensive and inadequate for charged-particle beams that can be manipulated using magnetic fields. Magnetic scanning has thus been introduced into ion therapy [4]. The target volume is dissected into slices of equal range and each slice is treated independently by scanning over the slice; once one slice has been treated, the beam energy and consequently the particle range is reduced and the next slice is treated.

Depending on the target geometry, parts of the tumour are pre-irradiated during the treatment of outer regions. The additional irradiation needed to achieve a homogeneous biological effect over the entire treatment volume must thus be distributed inhomogeneously over a given slice. Two strategies have been discussed, namely pixel scanning and raster scanning [6], involving discrete or

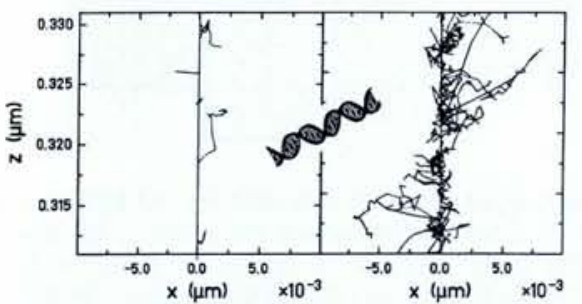

Fig. 2 - The tracks for protons (left) and carbon ions (right) compared with the dimensions of a DNA molecule shown schematically at the centre. The tracks were measured in water using $1 \mathrm{MeV}$ per nucleon projectiles. Carbon ions have a much higher probability to produce breaks simultaneously in two opposite strands of DNA [3].

Fig. 3 - A schematic illustration of the intensity controlled magnetic scanning system. The beam is guided by two magnets and the beam intensity is measured with a transmission counter. The beam path for each slice through the volume to be treated is dissected into 16000 points and the writing velocity (up to $1 \mathrm{~m} / \mathrm{s}$ ) of the beam is controlled using an ionization detector.

Fig. 4 - Simulating conformal irradiation of a tumour. The photograph corresponds to the three-dimensional distribution of dose given to a $60 \mathrm{~mm}$ in diameter spherical volume of water acting as a phantom. The $5 \mathrm{~mm}$ full-width half-maximum beam entering from the left was scanned using a precalculated pattern of 50-1500 pixels per slice at 30 different ranges to achieve an homogeneous dose distribution. The dose was measured using a stack of sheets of transparent nuclear track material. After irradiation, the stack was etched to give
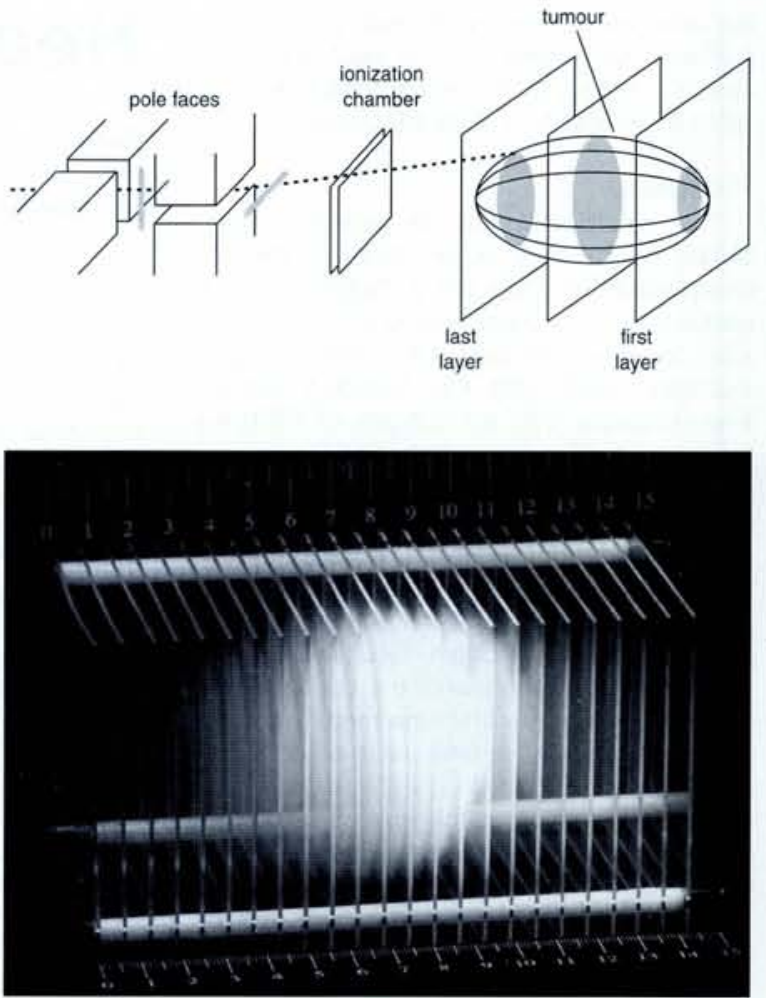

holes corresponding to tracks which scattered light to give a measure of the radiation damage.

continuous motion, respectively, of the beam. The differences are largely academic and essentially revolve around the issue of whether or not the beam needs to be turned off while moving from one point to the next. In practice, the beam diameter is several millimetres so if the distance between two pixels is small compared to the diameter, the beam need not be interrupted on moving from pixel to pixel (overexposure at a pixel can be largely compensated during irradiation at the next one). GSI has set up a digitized version of an intensity controlled, raster scanning system (Fig. 3). The use of a transmission counter to control the writing velocity has been demonstrated to be a very elegant and efficient method for conform treatment and for coping with fluctuations in the beam intensity. In preliminary experiments, a spherical volume of water has been successfully irradiated to a constant dose (Fig. 4)

\section{Computer Modelling}

The extended region of high biological efficiency in the Bragg region for ions such as carbon and oxygen will be restricted to the target volume using the novel technique of fast magnetic scanning with active energy variation. While this will open up new opportunities in conformal tumour therapy, it involves a quantum leap in the complexity of beam control and treatment planning. This is because the dose at various points in the target volume comprises a mixture of doses originating from the plateau and Bragg peak ions having very different biological efficiencies, depending on the atomic number and beam energy. A model [6] for the biological action is essential as it is impossible to measure all the necessary data. It is only because of a deeper understanding of physical and biological properties of charged particles that it will be possible to use focussed ion beams in the same way as a surgeon uses a scalpel.

\section{The GSI Therapy Unit}

Tumour conform treatment has not been implemented up to now owing to the poor time-structure of extracted beams coming from accelerators designed for physics experiments. Indeed, heavy particles such as neon ions were found to give no significant advantages over protons. The passive methods of beam shaping adopted from X-ray therapy which were used did not allow the irradiation to completely conform to a tumour so the maximum dose necessary for controlling the tumour could not be specified. A new facility at GSI plans to eliminate these shortcomings in treating deep-seated, inoperable cancer tumours by combining the novel technique of three-dimensional tumour conforming irradiation with the optimization of biological advantages using computer modelling. The Heavy-Ion Therapy Unit at GSI (HITAG: see insert), which was strongly endorsed by Dr. P. Krüger, Germany's science minister, during a visit to GSI last month, will use ions between carbon and oxygen at energies of 300-500 MeV per nucleon coming from the heavy-ion synchrotron SIS that can accelerate all particles from protons up to uranium ions to $1 \mathrm{GeV}$ per nucleon or more. A longstanding research programme at GSI has already characterised the beam structure and properties and acquired an extensive understanding of radiobiological aspects.

[1] Kraft G., Strahlenther. Onkol. 166 (1990) 10. [2] Enghardt W. et al., Phys. Med. Biol. 37 (1992) 2127

[3] Krämer G. \& Kraft G., in Biophysical Modelling of Radiation Effects, Eds: K.H. Chadwick et al. (Bristol, Philadelphia \& New York, 1992).

[4] Haberer Th. et al., Nucl. Inst. \& Methods A 330 (1993) 291.

[6] Farley F.J.M. \& Mandrillon P., Europhys. News 23 (1992) 3.

[6] Scholz M. \& Kraft G., in [3] 